\title{
1 A \\ Efficient Asymmetric Synthesis of Lamivudine and its enantiomer via Enzymatic Dynamic Kinetic Resolution
}

\author{
Lei Hu, Fredrik Schaufelberger, Yan Zhang and Olof Ramström*
}

\author{
KTH - Royal Institute of Technology, Department of Chemistry, Teknikringen 30, S- \\ 10044 Stockholm, Sweden.
}

\section{*ramstrom@kth.se}

Keywords: Dynamic kinetic resolution, Enzyme, Nucleoside, Asymmetric synthesis

\section{INTRODUCTION}

In the last decade, lamivudine has been one of the most successful drugs for the treatment of HIV as well as chronic Hepatitis B. It inhibits both type 1 and 2 of the human HIV reverse transcriptase and also the reverse transcriptase of hepatitis $\mathrm{B}$ in vitro. ${ }^{1}$ Although there are many reported syntheses for the isomerically pure lamivudine, most of them introduce the chiral 1,3-oxathiolane motif by either crystallization of the correct isomer from a racemic mixture, or by enzymatic hydrolysis/acetylation of the other stereoisomers. The main drawback of those methods is the great loss in yield, as no more than $50 \%$ yield could be obtained while maintaining high stereoselectivity, due to the nature of the kinetic resolution.

\section{RESULTS AND DISCUSSION}

In this study, by using surfactant-treated subtilisin Carlsberg (STS) as a green catalyst, intermediate 1 could be obtained in good yield and ee, and later converted to lamivudine after Vorbrüggen coupling and hydrolysis. The stereochemical configuration of the intermediates could be easily controlled as enantiomer 2 was also accessed by changing STS to Candida antarctica lipase $B(C A L B)$ under the same reaction conditions. Thus, this study describes an efficient methodology for the synthesis of 1,3oxathiolane-based nucleosides.

\section{CONCLUSION}

In summary, we have developed a three-step asymmetric synthesis of lamivudine using STS. This strategy represents a first entry to efficient highenantiopurity nucleoside analog synthesis. We have also described that the stereochemistry of the target molecules could be well controlled, as different isomers of the nucleoside intermediates were selectively obtained using different enzymes. This study thus offers a valuable methodology for the asymmetric synthesis of lamivudine as well as other nucleosides such as emtricitabine and apricitabine, where specific stereoisomers are desired.

\section{ACKNOWLEDGEMENTS}

This work was in part supported by the Swedish Research Council and the Royal Institute of Technology. LH and YZ thank the China Scholarship Council for special scholarship awards.

\section{REFERENCES}

${ }^{1}$ Romeo, G.; Chiacchio, U.; Corsaro, A.; Merino, P. Chem. Rev.2010, 110, 3337 .

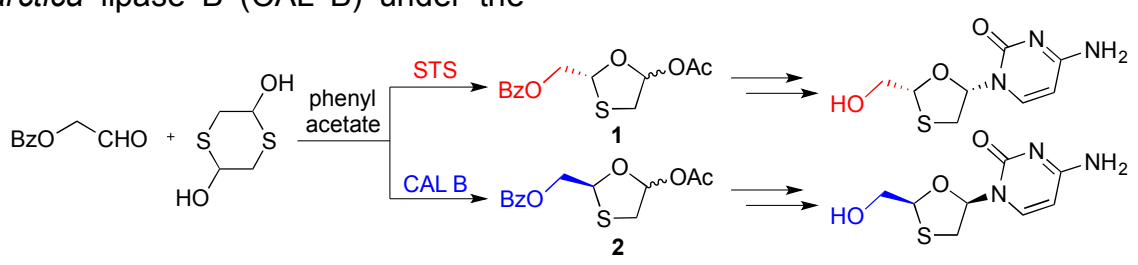

